\title{
Genomic aberrations in mucinous tubular and spindle cell renal cell carcinomas
}

\author{
Petter Brandal ${ }^{1}$, Agnes Kathrine Lie $^{2}$, Assia Bassarova ${ }^{2}$, Aud Svindland ${ }^{3}$, Bjørn Risberg², \\ Håvard Danielsen ${ }^{4}$ and Sverre Heim ${ }^{1,5}$ \\ ${ }^{1}$ Department of Cancer Genetics, The Norwegian Radium Hospital, Oslo, Norway; ${ }^{2}$ Department of Pathology, \\ The Norwegian Radium Hospital, Oslo, Norway; ${ }^{3}$ Department of Pathology, Aker University Hospital, Oslo, \\ Norway; ${ }^{4}$ Department of Medical Informatics, The Norwegian Radium Hospital, Oslo, Norway and ${ }^{5}$ Medical \\ Faculty, University of Oslo, Oslo, Norway
}

\begin{abstract}
Mucinous tubular and spindle cell carcinoma of the kidney is a new diagnostic entity. We present the pathologic and genomic characteristics of three such low-malignant tumors. Two of the tumors were found in women aged 19 and 52 years, the third tumor was found in an 80-year-old man, and the tumor stages were pT2NOMX, pT2NXMX, and pT1NXMX, respectively. Findings by immunohistochemistry were similar but not identical for the three cases; markers for both proximal and distal parts of the nephron were expressed in each tumor, a finding that is in agreement with data from previous studies. The Ki-67-labeling index was below 5 in all three cases. Two of the tumors were predominantly hypodiploid (DNA-indexes 0.77 and 0.80 ), whereas the third tumor was hypertriploid (1.57) as measured by DNA-image cytometry. From the latter tumor live cells were available making it possible to establish its karyotype: $62-70, X X X,+\operatorname{del}(X)(q 11),-1,+2,+4,-5,-6,+7,-8,-9,-10,-11$, $+12,-13,-14,-15,+16,+17,+18,-19,+20,+21,-22[c p 15]$. Interphase fluorescence in situ hybridization analyses with centromere-specific probes for chromosomes $1,3,4,6,7,9,10,17,18,20$, and $X$ showed that the two hypodiploid tumors had disomic and monosomic chromosome populations, whereas the karyotyped, near-triploid tumor was dominated by trisomic chromosome populations. Comparative genomic hybridization analysis was normal for the karyotyped tumor but abnormal for the two others. We conclude that multiple numerical chromosome aberrations may be a feature of mucinous tubular and spindle cell carcinomas of the kidney, but beyond that no clear-cut karyotypic aberration pattern is so far discernible.

Modern Pathology (2006) 19, 186-194. doi:10.1038/modpathol.3800499; published online 21 October 2005
\end{abstract}

Keywords: kidney; mucinous tubular and spindle cell tumor; numerical chromosome aberrations

Mucinous tubular and spindle cell carcinoma of the kidney is a relatively new entity. In the late 1990s, several reports describing low-grade, unclassified renal cell carcinomas (RCC) exhibiting mucinous, tubular, and spindle cell features and minimal mitotic activity were published, ${ }^{1-4}$ culminating in the WHO acceptance of these tumors as a distinct and new subgroup of RCC. ${ }^{5}$ A recent publication reviews clinical, pathological, and genomic characteristics of this new tumor entity. ${ }^{6}$ Although almost half of the tumors have been large at diagnosis, the prognosis seems to be excellent, and contrary to what applies to RCC in general, ${ }^{7}$ these

Correspondence: Professor Dr S Heim, MD, PhD, Department of Cancer Genetics, The Norwegian Radium Hospital, Montebello, 0310 OSLO, Norway.

E-mail: sverre.heim@medisin.uio.no

Received 22 June 2005; revised and accepted 26 August 2005; published online 21 October 2005 tumors seem to be more common in women. ${ }^{8}$ Mucinous tubular and spindle cell carcinomas morphologically replicate the lower parts of the nephron or the loop of Henle and are of a remarkably low grade for a carcinoma with spindle cell features. Microscopically, cuboidal cells are arranged in cords and tubules, separated by a pale mucinous stroma and intermixed with spindle cell areas. There is no clear consensus on the degree of morphological variation that may be present in these tumors, but as a minimum any tumor belonging to this subgroup should contain a certain amount of tubules, spindle cells, and mucin. The immunohistochemical profile reported for these tumors has varied and thus provides only inconclusive evidence as to which part(s) of the nephron they originate from.

Comparative genomic hybridization (CGH) analysis of mucinous tubular and spindle cell carcinomas of the kidney has been performed in two studies, with a total number of 17 tumors analyzed. Rakozy 
et $a l^{9}$ reported losses of chromosomes $1,4,6,8,9$, $13,14,15$, and 22 in all six tumors analyzed, as well as loss of the X-chromosome in three of the tumors. Weber et $a l^{10}$ found more complex genomic changes in eight of their 11 tumors, whereas three were normal, but as they only analyzed two to three metaphases per tumor, the conclusions seem somewhat uncertain. In both studies, however, interphase fluorescence in situ hybridization (IP-FISH) analyses were said to confirm the findings by CGH.

We present here the first karyotypic description of a mucinous tubular and spindle cell carcinoma of the kidney, as well as CGH and IP-FISH findings together with DNA ploidy and proliferative (Ki-67) analysis of the same and two other RCC of this subtype. All three tumors were also extensively characterized immunohistochemically.

\section{Materials and methods}

\section{Pathologic Case Reports}

Case 1: A $12 \mathrm{~cm}$ in diameter tumor was detected in the left kidney of a 19-year-old woman. The tumor was solid with a yellow to gray cut surface that also showed focal hemorrhages, but no cysts. The tumor was well demarcated and there was no evidence of local spreading or metastasis.

Case 2: A well-circumscribed $5.5 \mathrm{~cm}$ in diameter tumor was detected in the right kidney of an 80-yearold man. The cut surface of the tumor was light in color and had no cysts. There was no evidence of local spreading or metastasis.

Case 3: A $9.5 \mathrm{~cm}$ in diameter tumor was detected in the right kidney of a 52-year-old woman. The tumor was well delineated. The cut surface was partly white and partly yellow with no cysts, and there were no signs of locally infiltrative growth or distant metastasis.

The histologic examination in all three cases revealed both tubular and spindle cell areas with a mucinous stroma (Figure 1). The nuclei of the tumor cells were round and centrally located with small- to medium-sized nucleoli. There was no significant atypia. Mitoses and vascular invasion were not found.

\section{Immunohistochemistry}

Immunostaining for low molecular weight cytokeratin (CK) 7, high molecular weight CK (CK19 and $34 \beta$ E12), epithelial membrane antigen (EMA), vimentin, markers for proximal tubuli differentiation (CD10, CD15, RCC), villin, monoclonal carcinoembryonic antigen (mCEA), and the proliferative marker Ki-67 was performed in all three cases. The antibodies used and the immunostaining conditions are listed in Table 1. Formalin-fixed, paraffinembedded (FFPE) tissue sections $(4 \mu \mathrm{m})$ were mounted on super frost slides/plus slides and air dried for $24 \mathrm{~h}$ at $37^{\circ} \mathrm{C}$. After deparaffinization, heatinduced epitope retrieval was performed, and the EnVision Immunodetection System (DAKO, Glostrup, Denmark) was then used for detection. The antigen-antibody-enzyme complex was visualized by diaminobenzidine. Sections were counterstained with hematoxylin. Appropriate negative and positive controls were used in each staining run. The immunohistochemical reaction was evaluated semiquantitatively.

\section{DNA Content Measurements}

Procedures for measurement of DNA content and the criteria for ploidy classification were as described earlier, but with minor modifications. ${ }^{11}$ From all three tumors two $50-\mu \mathrm{m}$ sections were cut and enzymatically digested (SIGMA protease, type XXIV; Sigma Chemical Co., St Louis, MO, USA) for the preparation of monolayers. The DNA content of at least 1000 Feulgen-Schiff-stained nuclei was measured and analyzed using the Fairfield DNA Ploidy System (Fairfield Imaging Ltd, Kent, UK) and assessed according to an established protocol. A Zeiss Axioplan II microscope (Zeiss, Oberkochen, Germany) $(\times 40 / 0.65)$ with a 546-nm green filter was used for the analysis of the monolayers. At least 1000 epithelial cell nuclei were measured and stored in galleries for each case, with plasma cells and lymphocytes included as internal (DNA diploid) controls in all three cases.

\section{Cell Culturing and Karyotyping}

Fresh material was available only from tumor 1, partly as a preoperative biopsy and partly postoperatively from the surgically removed tumor. The tumor samples were manually minced and treated with collagenase, hyaluronidase, and neuraminidase until a suitable suspension of cells and cell clumps was obtained. After 5-7 days culturing in different flasks in a medium consisting of RPMI1640, 13\% fetal calf serum, and antibiotics, colchicine was added for the last $4 \mathrm{~h}$ and the short-term cultures were harvested according to standard protocols. ${ }^{12}$ The chromosomes in the dividing cells were then G-banded and a karyotype established in accordance with the recommendations of An International System for Human Cytogenetic Nomenclature. ${ }^{13}$

\section{Multiplex-FISH (M-FISH)}

Briefly, the newly dropped and pretreated chromosome preparations were denatured in 70\% formamide at $72{ }^{\circ} \mathrm{C}$ for $2 \frac{1}{2} \mathrm{~min}$, dehydrated in an ethanol series, and air dried before application of the SpectraVision probe (Vysis, Downers Grove, Illinois, USA) that had been denatured for $5 \mathrm{~min}$ at $72^{\circ} \mathrm{C}$. ${ }^{14}$ 

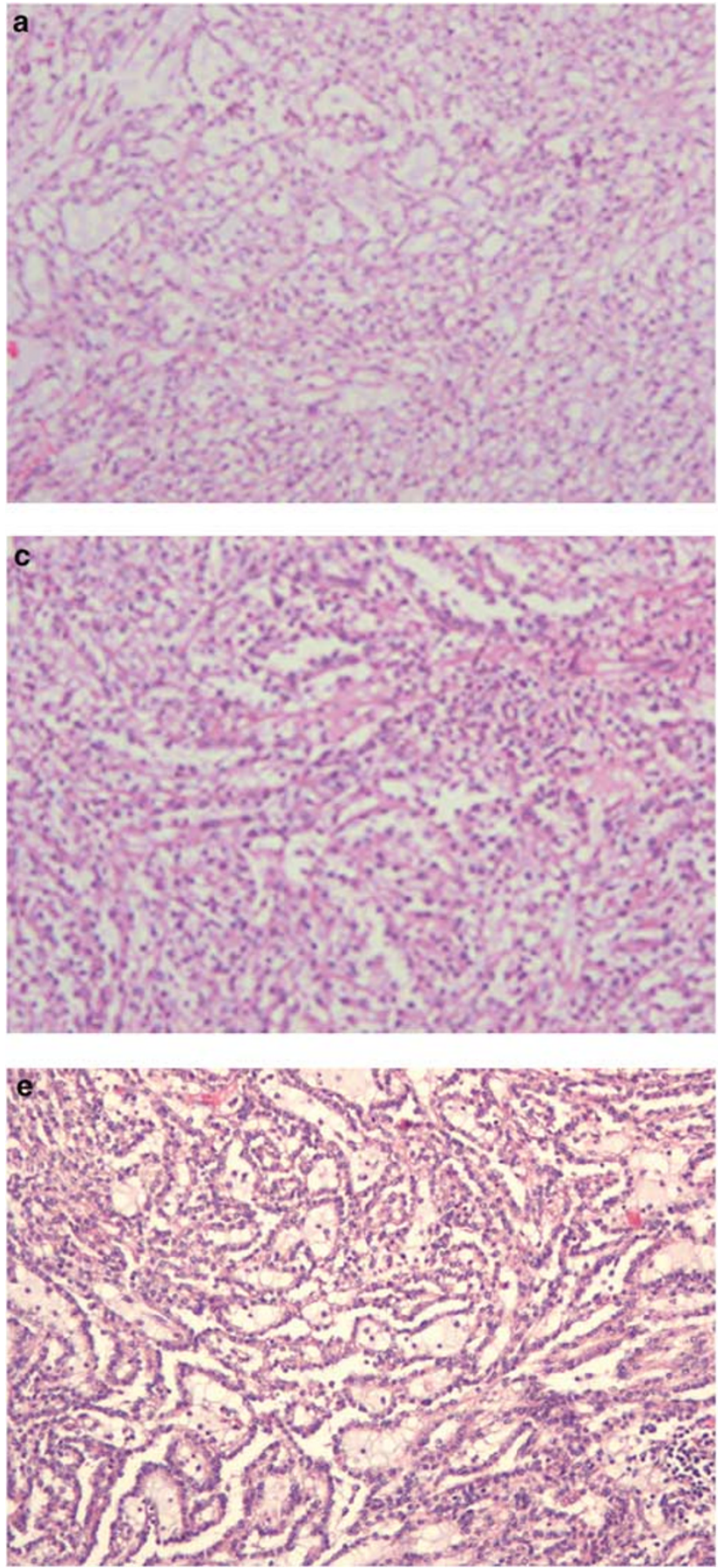
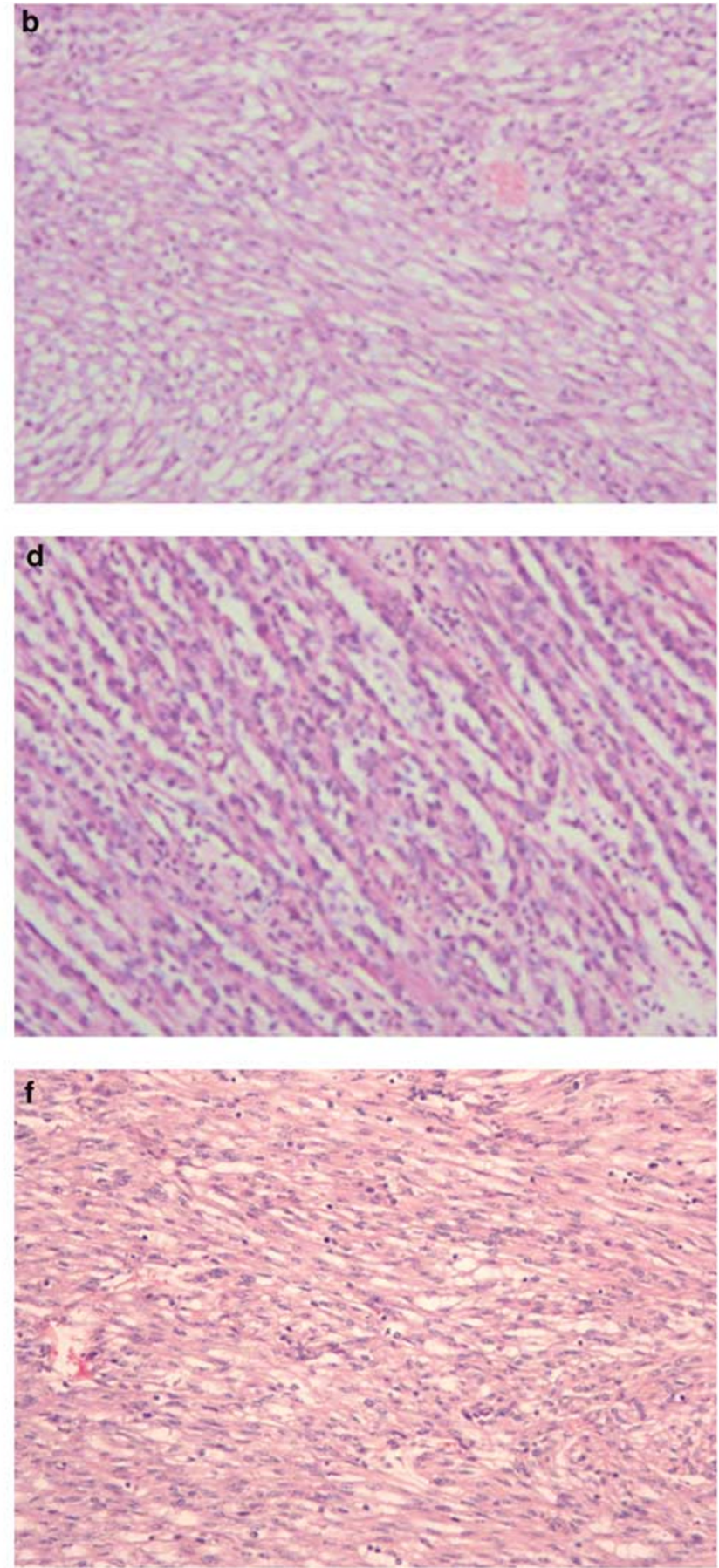

Figure 1 Light microscopic characteristics of the three mucinous tubular and spindle cell carcinomas showing typical morphology with small, elongated tubules separated by a pale mucinous stroma and areas with spindle cell configuration. All pictures are H\&E,

$\times 10$ magnification. (a and $\mathbf{b})$ are from tumor 1, (c and $\mathbf{d})$ are from tumor 2, (e and $\mathbf{f}$ ) are from tumor 3 .

The slides were incubated for 3 days in a moist chamber before posthybridization washes with amplification of the spectrum green signal, and then counterstained with DAPI. Fluorescent signals were detected and captured using Cytovision hardware and software, and image analysis was performed using the composite image of the five fluorophores and computerized pseudocoloring of the chromosomes.

\section{Comparative Genomic Hybridization}

DNA was isolated by the phenol-chloroform method as previously described, ${ }^{15}$ and metaphase target slides were prepared by dropping fixed cells from short-term cultured peripheral blood onto moist slides. The evaluation of the preparations was based on chromosomal length, color, degree of overlapping, amount of cytoplasm, and number of 
Table 1 Antibodies used and immunostaining conditions

\begin{tabular}{|c|c|c|c|}
\hline $\begin{array}{l}\text { Antibody } \\
\text { (clone) }\end{array}$ & Source & Dilution & HIER \\
\hline Cytokeratin 7 & Dako, Glostrup, Denmark & $1: 200$ & A \\
\hline Cytokeratin 19 & Dako, Glostrup, Denmark & $1: 50$ & A \\
\hline $34 \beta \mathrm{E} 12$ & Dako, Glostrup, Denmark & $1: 10$ & A \\
\hline EMA & Dako, Glostrup, Denmark & $1: 40$ & B \\
\hline Vimentin & Dako, Glostrup, Denmark & $1: 50$ & A \\
\hline CD10 & $\begin{array}{l}\text { Novocastra, Newcastle upon } \\
\text { Tyne, UK }\end{array}$ & $1: 40$ & A \\
\hline CD15 & Dako, Glostrup, Denmark & $1: 10$ & B \\
\hline Villin & Immunotech & $1: 25$ & A \\
\hline RCC & $\begin{array}{l}\text { Novocastra, Newcastle upon } \\
\text { Tyne, UK }\end{array}$ & $1: 20$ & B \\
\hline CEA & Dako, Glostrup, Denmark & $1: 100$ & B \\
\hline Ki-67 (MIB-1) & Dako, Glostrup, Denmark & $1: 150$ & A \\
\hline
\end{tabular}

HIER, heat-induced epitope retrieval; A, $4 \times 5$ min TRIS/EDTA buffer; $\mathrm{B}, 4 \times 5$ min low $\mathrm{pH}$ buffer.

mitoses. ${ }^{16}$ Slides were stored at room temperature for 1-4 weeks before use. CGH was performed on FFPE material from all three tumors as previously described, ${ }^{17-19}$ and the slides were examined in a fluorescence microscope (Zeiss Axioplan, Oberkochen, Germany). Single-color images (FITC, TexasRed, and DAPI) of metaphase spreads were sequentially photographed with a Cohu 4900 CCD (12-bit gray scale) camera, using Cytovision hardware and software.

Chromosomes were karyotyped based on their inverted DAPI-banding appearance. Fluorescence ratio profiles (green to red) were calculated for individual chromosomes, data from 11 to 19 representative copies of each chromosome were combined, and average ratio profiles with $95 \%$ confidence intervals were calculated for each tumor. The centromeric and pericentromeric heterochromatic regions were not evaluated. We chose thresholds for loss and gain corresponding to loss or gain of one chromosome homologue in $50 \%$ of the cells analyzed, that is, 0.83 and 1.17, respectively, for the hypotriploid karyotyped mucinous tubular and spindle cell carcinoma and 0.75 and 1.25 for the two hypodiploid tumors. Appropriate positive and negative controls were included in all experiments.

\section{Interphase Fluorescence In Situ Hybridization}

Single- and dual-color IP-FISH with probes (alphasatellite and satellite II/satellite III) specific for the centromeres of chromosomes 1, 3, 4, 6, 7, 9, 10, 17, 18,20 , and $\mathrm{X}$ was performed on extracted nuclei. These probes were chosen because the chromosomes they react with have been reported to be aberrant in mucinous tubular and spindle cell carcinoma earlier $^{9,10}$ and/or were involved in abnormalities in our karyotyped case. Directly labeled fluorescent probes and appropriate hybridization and counterstaining solutions were supplied by Vysis and
Q-BIOgene (AH diagnostics, Oslo, Norway). Interphase nuclei were extracted from areas with almost exclusively tumor cells, including both spindle cell and tubular areas, by a method modified from Hedley and Liehr, ${ }^{15,20}$ and the resulting preparations were pretreated as described earlier with but minor modifications. ${ }^{15,21}$ Following hybridization procedures that have been described, ${ }^{15} 200$ successive, whole, and single nuclei were examined in a Zeiss fluorescence microscope and images were captured using a Cohu camera. The specificity of the probes was validated by FISH experiments on slides with metaphases and interphase nuclei from a karyotypically normal person. A minimum of $5 \%$ of the nuclei had to show a certain number of signals for each probe if the corresponding disomy or aneusomy for that probe should be accepted to be present in a population of the cells counted. This $5 \%$ limit was chosen based on the findings on our control slides.

\section{Results}

\section{Immunohistochemistry}

Vimentin was diffusely expressed in all three cases. Focal or diffuse positivity for CK7 and CK19 was observed in all tumors, while $34 \beta \mathrm{E} 12$ was detected only in the tubular areas of tumors 2 and 3. EMA was also focally positive in those two cases. The renal proximal tubular brush border marker RCC was expressed focally only in the tubular component in all the cases, whereas the other two proximal tubular markers, CD15 and CD10, were negative in tumors 1 and 3 and in tumor 2, respectively. The tumors did not express villin or mCEA. Ki-67labeling index was below $5 \%$ in all three cases (Table 2, Figure 2).

\section{DNA Content Measurements}

All three tumors were aneuploid with at least two ploidy peaks (Figure 3). Tumor 1 had a dominant hypertriploid peak (DNA-index 1.57), whereas tumors 2 and 3 both had hypodiploid clones dominating ( 0.80 and 0.77 , respectively), as well as smaller hypertriploid peaks. The samples all had a diploid peak as the second largest peak.

\section{Karyotyping}

The karyotype of the one mucinous tubular and spindle cell carcinoma from which live cells were available for in vitro culturing (tumor 1), was: $62-70, X X X,+\operatorname{del}(\mathrm{X})(\mathrm{q} 11),-1,+2,+4,-5,-6,+7,-8$, $-9,-10,-11,+12,-13,-14,-15,+16,+17,+18,-19$, $+20,+21,-22[\mathrm{cp} 15]$ (Figure 4). The structurally changed chromosome was initially thought to be a $\operatorname{del}(11)(q 13)$, but the M-FISH analysis showed it to be a $\operatorname{del}(\mathrm{X})(\mathrm{q} 11)$. The karyotypic findings from the 
Table 2 Immunohistochemical profile of the three mucinous tubular and spindle cell renal cell carcinomas

\begin{tabular}{|c|c|c|c|c|c|c|}
\hline \multirow{2}{*}{$\begin{array}{l}\text { Epitope } \\
\text { CK7 }\end{array}$} & \multicolumn{2}{|c|}{ Tumor 1} & \multicolumn{2}{|c|}{ Tumor 2} & \multicolumn{2}{|c|}{ Tumor 3} \\
\hline & $+/-$ & Focal & +++ & Diffuse & + & Focal \\
\hline CK19 & ++ & Focal & +++ & Diffuse & ++ & Focal \\
\hline $34 \beta \mathrm{E} 12$ & - & & ++ & Focal, tubular & \pm & Focal, tubular \\
\hline EMA & - & & ++ & Focal & \pm & Focal \\
\hline Vimentin & +++ & Diffuse & +++ & Diffuse & ++ & Diffuse \\
\hline CD10 & + & Focal & - & & \pm & Focal \\
\hline CD15 & - & & + & Focal, tubular & - & \\
\hline RCC & ++ & Focal, tubular & ++ & Focal, tubular & ++ & Focal, tubular \\
\hline Villin & - & & - & & - & \\
\hline mCEA & - & & - & & - & \\
\hline Ki-67 & $<5 \%$ & & $<5 \%$ & & $<5 \%$ & \\
\hline
\end{tabular}

preoperative biopsy and the operation specimen were identical.

\section{Comparative Genomic Hybridization}

The analysis was successful for all three tumors. Tumor 1 showed no copy number changes, whereas in tumor 2 some, and in tumor 3 multiple copy number aberrations were found, both losses and gains in both tumors (Table 3, Figure 4). There were three areas of common gains in the two abnormal tumors, corresponding to $2 \mathrm{p} 16-\mathrm{p} 13,2 \mathrm{q} 22-\mathrm{q} 33$, and 5 12. No common lost regions were identified.

\section{Interphase Fluorescence In Situ Hybridization}

Chromosomes 1, 3, 4, 6, 7, 9, 10, 17, 18, 20, and X were analyzed for all three tumors (Tables 3 and 4 , Figure 4). For tumor 1, each probe had three signals in the majority of the nuclei (58-72\%) and two signals in $27-34 \%$ of the nuclei. Chromosomes 1,4 , 7, 17 , and 20 also had a small but significant proportion (9-16\%) of the nuclei displaying four signals. In tumor 2 , the probes for chromosomes 1 , 4,6 , and $X$ had one signal in each nucleus as the major finding (54-90\%), whereas for the rest of the examined chromosomes, two signals per nucleus dominated (57-97\%). The findings in tumor 3 showed some similarity to those in tumor 2: Probes specific for chromosomes 1, 3, 6, and 9 showed one signal in $63-70 \%$ of the nuclei, whereas the other probes gave two signals in $50-100 \%$ of the nuclei.

\section{Discussion}

The three tumors in this series all exhibited histological characteristics sufficient for the diagnosis of mucinous tubular and spindle cell carcinoma of the kidney. Both tubular and spindle cells with minimal or no atypia were seen embedded in a myxoid stroma. ${ }^{5}$ The tumors were well delineated and showed no signs of invasion into the renal pelvis or through the renal capsule.
In all three tumors, the percentage of Ki-67positive cells was below five, suggesting a low proliferative activity, a finding that may in part explain the low malignancy of this tumor type. The immunohistochemical profile was not consistent; all three tumors were positive for markers of the proximal tubuli as well as for markers for the distal part of the nephron. Similar variability has been observed also in previous studies. ${ }^{8}$ Based on the extensive immunophenotypic variation observed, one may speculate that the cell of origin in these tumors is pluripotent and differentiates in different directions based on sequentially acquired genomic abnormalities.

The findings by DNA-ploidy analysis were of considerable interest. Tumors 2 and 3 both had hypodiploid clones dominating, with DNA indexes of 0.80 and 0.77 , respectively, but they also had diploid and very small hypertriploid clones. These near-triploid cells could be endoreduplicated cells from the hypodiploid population. The proliferative activity as evaluated from the S-phase fraction of the DNA histograms was small, in agreement with the Ki-67 findings. The DNA indexes were in accordance with the findings by IP-FISH, and they are also important for the interpretation of the CGH investigations (see below). Tumor 1 had, in addition to a small diploid clone, a larger cell population with a DNA-ploidy peak of 1.57 , which is about the double of the DNA-ploidy levels for tumors 2 and 3. Maybe this could be the result of duplication of the DNA content in an earlier stage hypodiploid cell, giving rise to a cell favored by selection.

Tumor 1 is the first karyotyped mucinous tubular and spindle cell carcinoma of the kidney. Mostly numerical chromosomal aberrations were detected, with gain or loss of multiple whole chromosomes compared with $3 \mathrm{n}$, in addition to one structural change believed to be a deletion of most of the long arm of one copy of the X-chromosome. IP-FISH also showed that the tumor was predominantly triploid for all investigated chromosomes, although smaller diploid and even tetraploid populations were present as well. The IP-FISH findings thus agreed totally with the karyotypic findings in this tumor. 

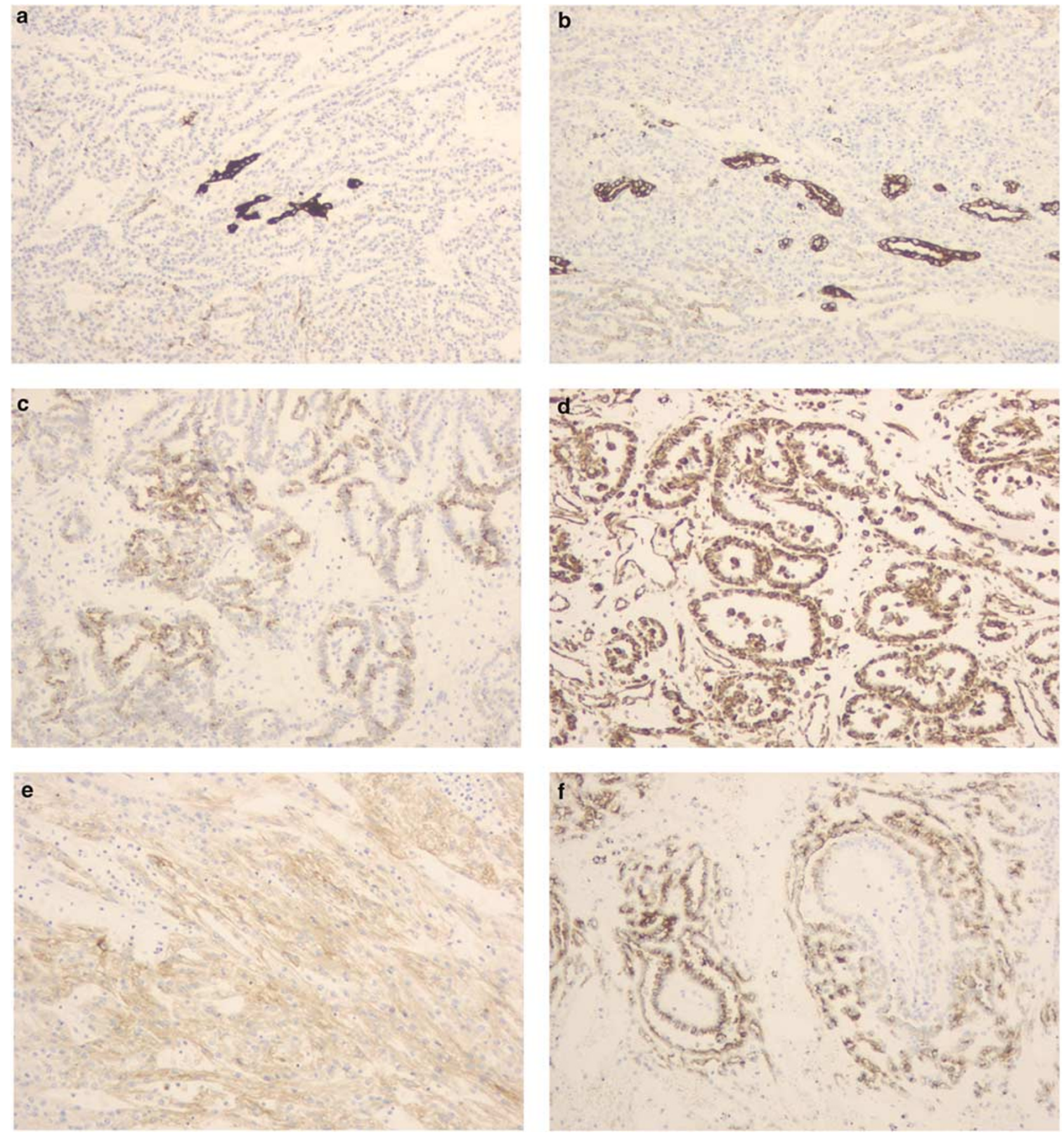

Figure 2 Immunohstochemical profile of the mucinous tubular and spindle cell carcinoma of tumor 3. (a) Focal positivity for cytokeratin 7; (b and c) Focal immunoreactivity for cytokeratin 19; (d) Diffuse strong positive reaction for vimentin; (e) Weak focal CD10 expression; (f) Positive reaction with RCC antibody in areas simulating immature glomerular structures (EnVision, $\times 10$ ).

The IP-FISH analyses in the two other tumors showed that either one or two copies of the chromosomes probed were found, but again the observed combination of monosomies and disomies corresponded well with the DNA-ploidy measurements.

CGH was normal in tumor 1, the only karyotyped tumor, six copy number aberrations were observed in tumor 2 , whereas tumor 3 was highly abnormal.
The scored copy number aberrations affected small genomic areas in tumor 2, but many of the chromosomes deviated towards loss or gain of the whole chromosome, although not enough to exceed the threshold value. A similar situation with individual whole chromosome profiles deviating towards gain or loss could be detected also in tumor 3 , but with the difference that in tumor 3 many of the deviations were scorable. The whole of chromosomes 10, 16, 

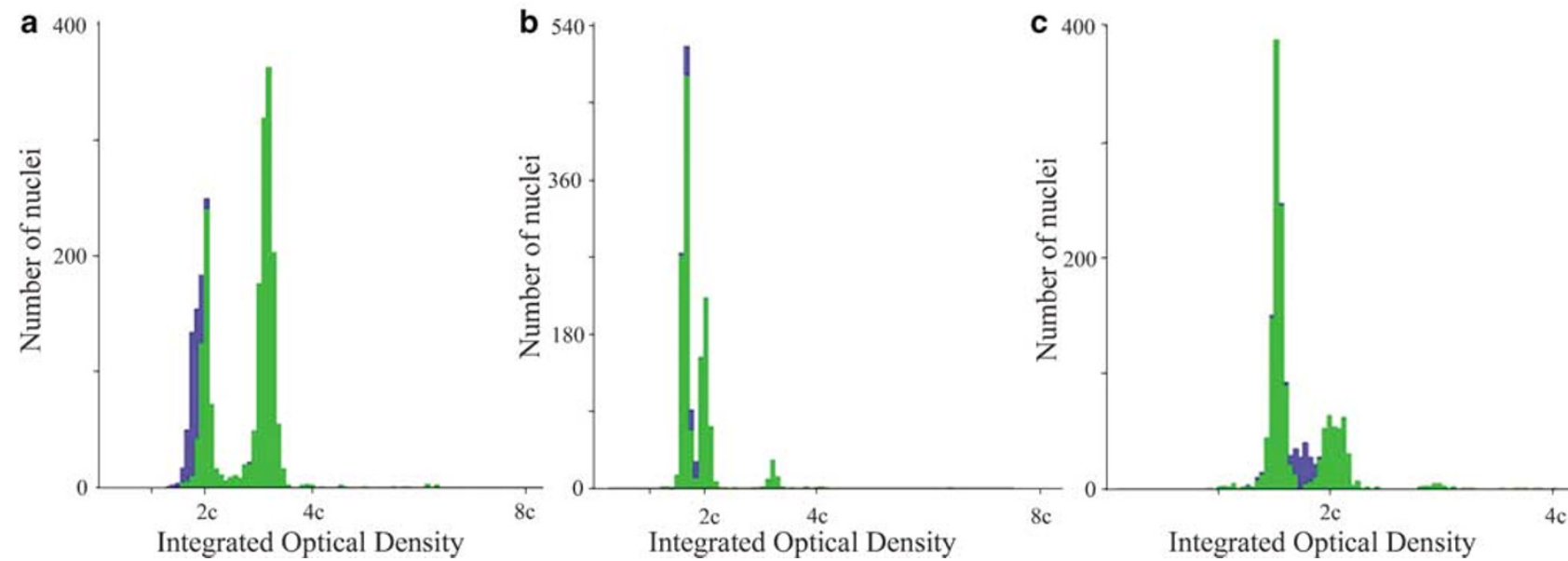

Figure 3 Ploidy distribution for the three mucinous tubular and spindle cell carcinomas of the kidney. The green area shows the distribution for tumor cells, the blue area marks the distribution for the internal control cells (plasma cells and lymphocytes). (a) Tumor 1; (b) Tumor 2; (c) Tumor 3.

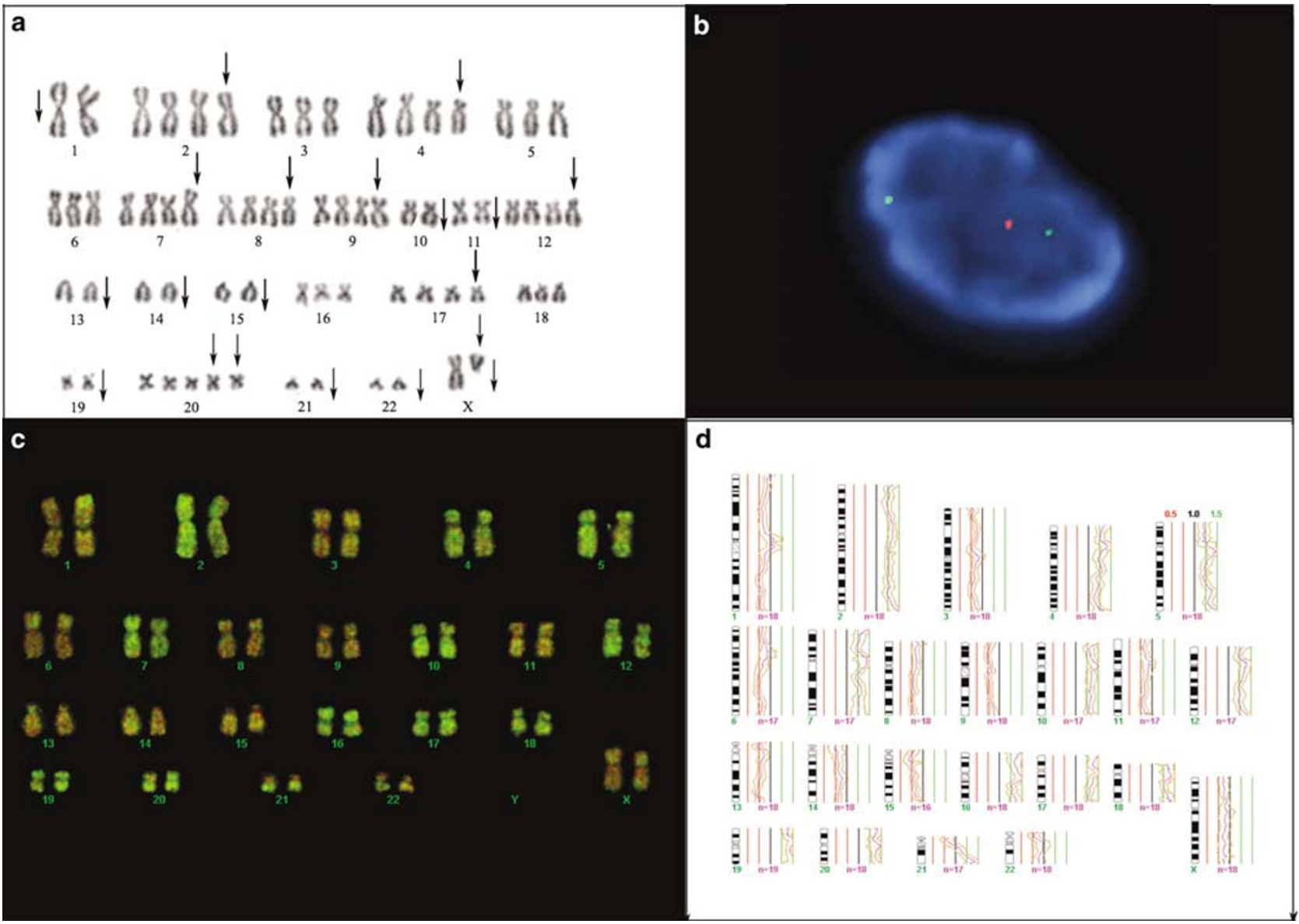

Figure 4 (a) Karyogram from a cell of tumor 1, displaying multiple numerical chromosome abnormalities as well as one structural aberration, $\operatorname{del}(\mathrm{X})(\mathrm{q} 11)$. Arrows mark each aberration compared with the normal triploid level (3n); (b) Interphase-FISH picture of a cell from tumor 3 with two green dots (probe for chromosome 4) and one red dot (probe for chromosome 1); (c and d) CGH-metaphase and corresponding profile from tumor 3. Tumor-DNA labeled in green, reference-DNA labeled in red. The pink line in the profile represents the average tumor/reference ratio, whereas the yellow lines represent the limits for the $95 \%$ confidence interval.

$17,18,19,20$, and 21 were gained, most of chromosomes $2,5,7$, and 12 were also gained, whereas especially chromosomes $3,8,9$, and 13 were almost scorable as losses. It is important to remember that for a ploidy level between the haploid and diploid one, as was the case in tumors 2 and 3 , the 
Table 3 Clinical and cytogenetic characteristics of the three mucinous tubular and spindle cell carcinomas

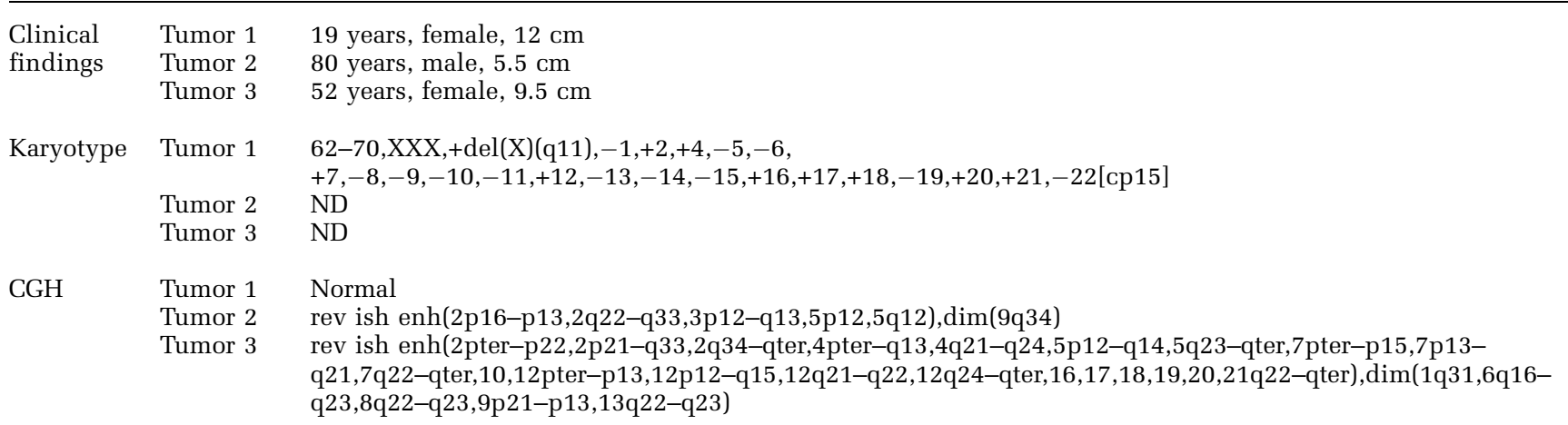

IP-FISH Chromosome and ploidy level ${ }^{\text {a }}$

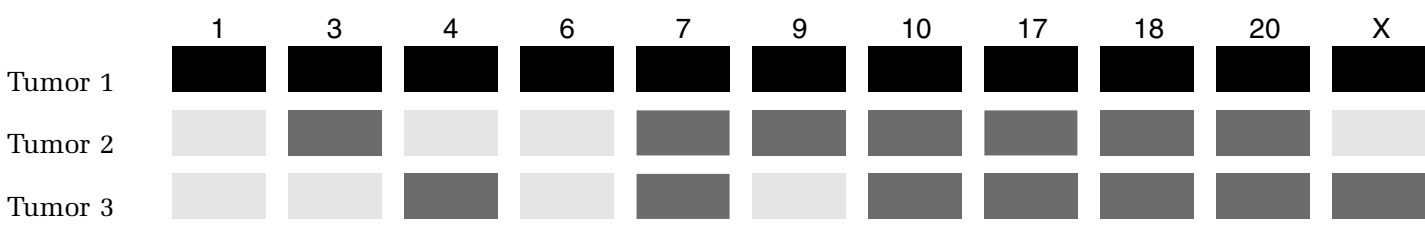

ND, not determined; CGH, comparative genomic hybridization; IP-FISH, interphase fluorescence in situ hybridization.

${ }^{\mathrm{a}}$ The dominant ploidy level for each chromosome is shown.

: monosomy; _ : disomy;

: trisomy.

Table 4 IP-FISH results for the three mucinous tubular and spindle cell carcinomas

\begin{tabular}{|c|c|c|c|c|c|c|c|c|c|c|c|c|}
\hline \multirow[t]{2}{*}{ Tumor no. } & \multicolumn{11}{|c|}{ Chromosome and signal frequencies } & \multirow[t]{2}{*}{ No. of signals per cell } \\
\hline & 1 & 3 & 4 & 6 & 7 & 9 & 10 & 17 & 18 & 20 & $X$ & \\
\hline \multirow[t]{4}{*}{ Tumor 1} & & & & & & & & & & & & 1 \\
\hline & 28 & 31.5 & 26.5 & 29.5 & 29.5 & 28 & 33.5 & 29.5 & 32 & 29.5 & 33.5 & 2 \\
\hline & 61.5 & 65 & 58 & 68 & 59 & 71.5 & 63.5 & 58 & 66 & 59 & 63.5 & 3 \\
\hline & 10.5 & & 15.5 & & 11.5 & & & 9 & & 11.5 & & 4 \\
\hline \multirow[t]{3}{*}{ Tumor 2} & 54.5 & 6.5 & 53.5 & 69.5 & 43 & & & & 7.5 & & 89.5 & 1 \\
\hline & 45 & 90.5 & 46.5 & 30.5 & 57 & 97 & 95.5 & 97 & 93 & 90.5 & 5.5 & 2 \\
\hline & & & & & & & & & & & & 3 \\
\hline \multirow[t]{3}{*}{ Tumor 3} & 62.5 & 69.5 & & 69.5 & & 69 & & & & & 49 & 1 \\
\hline & 37.5 & 30.5 & 98.5 & 30.5 & 98 & 31 & 99.5 & 97 & 95.5 & 74 & 50 & 2 \\
\hline & & & & & & & & & & 25.5 & & 3 \\
\hline
\end{tabular}

Only signal frequencies exceeding the threshold value of $5 \%$ are shown.

comparative nature of the CGH method represents an important methodological problem. ${ }^{22}$

In the few genomic investigations hitherto performed, based only on CGH and FISH, ${ }^{9,10}$ it has been suggested that mucinous tubular and spindle cell carcinomas of the kidney have a characteristic pattern of copy number aberrations as detected by CGH. ${ }^{5}$ These are losses of (or partly from) chromosomes $1,4,6,8,9,13,14,15$, and 22 , as well as gains of all or parts of chromosomes $7,11,16$, and 17. In the three tumors we examined, some of these changes were seen as were also other abnormalities. In one of the tumors (tumor 1), the mostly numerical changes found by karyotyping and IP-FISH were present only in a small proportion of cells and therefore not detectable by CGH. When assessing all available genomic information from previously performed studies as well as this one, we find that all chromosomes have been involved in aberrations in one way or another. The data are therefore too sparse and variable to conclude that mucinous tubular and spindle cell carcinomas of the kidney have a characteristic pattern of genomic aberrations separating them from other subgroups of RCC. Further (cyto)genetic studies on larger series of tumors should be performed, and whenever possible a combination of methods should be used to better characterize each tumor and to overcome the inherent weaknesses of all methods when used alone. It would also be of interest to microdissect the tubular 
and spindled elements and perform molecular cytogenetic analyses on these separately to see whether genomic differences can be found correlating with the phenotypic characteristics.

\section{Acknowledgement}

This work has been financially supported by The Norwegian Cancer Society through Grant number B94106/017.

\section{References}

1 Otani M, Shimizu T, Serizawa H, et al. Low-grade renal cell carcinoma arising from the lower nephron: a case report with immunohistochemical, histochemical and ultrastructural studies. Pathol Int 2001;51:954-960.

2 Hes O, Hora M, Perez-Montiel DM, et al. Spindle and cuboidal renal cell carcinoma, a tumour having frequent association with nephrolithiasis: report of 11 cases including a case with hybrid conventional renal cell carcinoma/ spindle and cuboidal renal cell carcinoma components. Histopathology 2002;41:549-555.

3 Lloreta J, Corominas JM, Munne A, et al. Low-grade spindle cell carcinoma of the kidney. Ultrastruct Pathol 1998;22:83-90.

4 He Q, Ohaki Y, Mori O, et al. A case report of renal cell tumor in a 45-year-old female mimicking lower portion nephrogenesis. Pathol Int 1998;48:416-420.

5 Srigley JR. Mucinous tubular and spindle cell carcinoma In: Eble JN, Sauter G, Epstein JI, Sesterhenn IA (eds). Pathology \& Genetics of Tumours of the Urinary System and Male Genital Organs 1st edn. IARC Press: Lyon, 2004, pp 40.

6 Kuroda N, Toi M, Hiroi M, et al. Review of mucinous tubular and spindle-cell carcinoma of the kidney with a focus on clinical and pathobiological aspects. Histol Histopathol 2005;20:221-224.

7 Meloni-Ehrig AM. Renal cancer: cytogenetic and molecular genetic aspects. Am J Med Genet 2002;115: 164-172.

8 Eble JN. Mucinous tubular and spindle cell carcinoma and post-neuroblastoma carcinoma: newly recognised entities in the renal cell carcinoma family. Pathology 2003;35:499-504.

9 Rakozy C, Schmahl GE, Bogner S, et al. Low-grade tubular-mucinous renal neoplasms: morphologic, immunohistochemical, and genetic features. Mod Pathol 2002;15:1162-1171.
10 Weber A, Srigley J, Moch H. Mucinous spindle cell carcinoma of the kidney. A molecular analysis. Pathologe 2003;24:453-459.

11 Sudbo J, Kildal W, Johannessen AC, et al. Gross genomic aberrations in precancers: clinical implications of a long-term follow-up study in oral erythroplakias. J Clin Oncol 2002;20:456-462.

12 Mandahl N. Methods in solid tumors cytogenetics In: Rooney DE, Czepulkowski BH (eds). Human cytogenetics-a practical approach, Vol II, Malignancy and Acquired Abnormalities. IRL Press: Oxford, 1992, pp 155-187.

13 Mitelman F (ed). ISCN. An International System for Human Cytogenetic Nomenclature. S. Karger: Basel, 1995.

14 Micci F, Teixeira MR, Heim S. Complete cytogenetic characterization of the human breast cancer cell line MA11 combining G-banding, comparative genomic hybridization, multicolor fluorescence in situ hybridization, RxFISH, and chromosome-specific painting. Cancer Genet Cytogenet 2001;131:25-30.

15 Brandal P, Bjerkehagen B, Heim S. Molecular cytogenetic characterization of tenosynovial giant cell tumors. Neoplasia 2004;6:578-583.

16 Karhu R, Kahkonen M, Kuukasjarvi T, et al. Quality control of CGH: impact of metaphase chromosomes and the dynamic range of hybridization. Cytometry 1997;28:198-205.

17 Kallioniemi A, Kallioniemi OP, Sudar D, et al. Comparative genomic hybridization for molecular cytogenetic analysis of solid tumors. Science 1992;258: 818-821.

18 Kallioniemi OP, Kallioniemi A, Piper J, et al. Optimizing comparative genomic hybridization for analysis of DNA sequence copy number changes in solid tumors. Genes Chromosomes Cancer 1994;10: 231-243.

19 Kraggerud SM, Szymanska J, Abeler VM, et al. DNA copy number changes in malignant ovarian germ cell tumors. Cancer Res 2000;60:3025-3030.

20 Hedley DW, Friedlander ML, Taylor IW, et al. Method for analysis of cellular DNA content of paraffinembedded pathological material using flow cytometry. J Histochem Cytochem 1983;31:1333-1335.

21 Knuutila S, Nylund SJ, Wessman M, et al. Analysis of genotype and phenotype on the same interphase or mitotic cell. A manual of MAC (morphology antibody chromosomes) methodology. Cancer Genet Cytogenet 1994;72:1-15.

22 Brandal P, Busund LT, Heim S. Chromosome abnormalities in juxtaglomerular cell tumors. Cancer 2005; 104:504-510. 